\title{
Community-based pharmacies: an opportunity to recruit patients?
}

\author{
Isabelle Peytremann-Bridevaux $\cdot$ Julie Bordet $\cdot$ Valérie Santschi • \\ Tinh-Hai Collet $\cdot$ Marc Eggli $\cdot$ Bernard Burnand
}

Received: 28 November 2011 / Accepted: 7 June 2012 / Published online: 1 July 2012

(C) Swiss School of Public Health 2012

Keywords Patients recruitment - Diabetes ·

Pharmacies roles

\section{Introduction}

Recruiting representative population groups with a specific disease is a challenge. In jurisdictions with no existing or no access to a database including individual disease diagnoses (e.g., electronic medical records), there are few satisfying strategies. Usually, population health surveys do not provide enough diagnostic information and may not allow recruiting large enough samples to study a specific disease. Registries or clinical cohorts may exist for specific diseases, but are often not truly population-based. Recruitment from patients' associations and physicians' practices may also be selective. In addition, the latter is difficult to run in busy primary care clinics. Finally, the type of information and the possibility to access health insurances' databases may preclude their use. For diseases that require treatment involving pharmacies regularly, we propose to recruit patients in community pharmacies; this

\footnotetext{
I. Peytremann-Bridevaux $(\bowtie) \cdot$ J. Bordet $\cdot$ V. Santschi .

B. Burnand

Institute of Social and Preventive Medicine (IUMSP),

Lausanne University Hospital, 10 Route de la Corniche,

1010 Lausanne, Switzerland

e-mail: Isabelle.Peytremann-Bridevaux@chuv.ch
}

\section{T.-H. Collet}

Department of Ambulatory Care and Community Medicine,

University of Lausanne, Lausanne, Switzerland

\section{Eggli}

Service of Endocrinology, Diabetes, and Metabolism,

Lausanne University Hospital, Lausanne, Switzerland has been seldom described (Knoester et al. 2005; Van Wieren-de Wijer et al. 2009). In the canton of Vaud, Switzerland ( $\sim 700,000$ residents), we have applied such an approach to conduct a survey designed to describe the current population of diabetic patients and to assess the quality of their care. This survey was developed within the framework of a regional diabetes program that started in the summer of 2010 (Hagon-Traub et al. 2010), as one of many such programs implemented to tackle the chronic diseases epidemic (Nolte et al. 2008).

The aim of this Hints and Kinks is to present a pharmacy-based approach of recruiting diabetic patients.

\section{Methods}

Taking into account the clustering of data by pharmacies (40 pharmacies recruiting each 15 patients, intra-class correlation 0.05 , alpha 0.05 , beta 0.2 ), 600 diabetic patients were considered enough to get a good precision around our primary outcomes (mean HbA1C, mean Physical and Mental summary score of the SF-12, \% of patients with specific annual checks).

To optimize the representativeness of the sample of diabetic patients, we decided to randomly select pharmacies. Expecting a participation rate of $50 \%$, we first chose 80 pharmacies out of the 254 ones registered in the canton in April 2011 (130 independent and 124 chain pharmacies). Since we did not reach our 40 target pharmacies, we redrew another sample of 60 . After this two-step procedure, 56 pharmacies accepted to participate. Both participating and non-participating pharmacies were asked to fill in a brief questionnaire on their practice characteristics (pharmacy type, pharmacy size and pharmacy location). Reasons for participation refusal were also asked. 
In September 2011, pharmacists recruited consecutive non-institutionalized adult diabetic patients ( $\geq 18$ years, type 1 or 2 diabetes, disease duration $\geq 12$ months) who were visiting the pharmacy with a prescription for oral antidiabetic drugs, insulin, glycemic strips or glucose meter. Women with gestational diabetes, patients not residing in the canton of Vaud, not speaking and understanding French well enough or presenting obvious cognitive impairment were excluded. The latter two eligibility criteria were subjectively assessed by the pharmacists. Age was recorded according to data on the prescription, and diabetes duration was asked to the patient. Physicians did not participate in the recruitment phase but were asked to fill in a short questionnaire (clinical and laboratory data), if patients did share their contact details.

A first letter was sent to all physicians and pharmacists of the canton, announcing the study. Another letter explaining the recruitment process to the randomly selected pharmacies was then sent. The recruitment was expected to last less than 10 min per patient, and pharmacies would receive a CHF 5 incentive for each questionnaire returned. Finally, we organized a meeting with pharmacists, to launch the study, answer final questions, and provide all needed documents; those who were not able to attend the meeting were seen in person during the same week. We contacted pharmacists weekly during the recruitment period. Since the patients' participation rate was lower than expected (i.e. less than 600 questionnaires sent back), the recruitment period was extended from 4 to 6 weeks. During the final phone call, main reasons for ineligibility, main barriers to recruitment, and main reasons for patients' refusal were investigated.

The protocol was approved by the Canton's Ethics Committee of Research on Human Beings.

\section{Results}

The characteristics of participating and non-participating pharmacies (Table 1) did not differ significantly except for size of the pharmacy. Lack of time $(68 \%)$ and not having enough diabetic patients $(23 \%)$ were the main reasons for refusing to participate.

During the recruitment period, 809 of 1,013 eligible patients accepted to receive the questionnaire from a pharmacy (Fig. 1); approximately $17 \%$ of approached patients (0-52\% depending on pharmacy) were ineligible. Finally, 406 patients returned the paper-questionnaire, leading to an estimated patients' participation rate of $50.2 \%(406 / 809)$. At the end of the 6-week period, main reported barriers for recruitment were ineligibility of patients $(34 \%)$, duration of recruitment period too short (25\%), difficulty to propose the study to patients $(25 \%)$, and difficulty to motivate the pharmacy's team (14\%).
Table 1 Characteristics of randomly selected $(n=140)$ participating and non-participating pharmacies of the canton of Vaud (Switzerland, 2011)

\begin{tabular}{|c|c|c|c|}
\hline & $\begin{array}{l}\text { Participating } \\
\text { pharmacies } \\
(n=56)\end{array}$ & $\begin{array}{l}\text { Non- } \\
\text { participating } \\
\text { pharmacies }^{\mathrm{a}} \\
(n=47)\end{array}$ & $\begin{array}{l}\text { All } \\
\text { pharmacies } \\
(N=254)\end{array}$ \\
\hline \multicolumn{4}{|l|}{ Pharmacy type } \\
\hline Independent & $37(66 \%)$ & $29(62 \%)$ & $130(51 \%)$ \\
\hline $\begin{array}{l}\text { Chain (Sunstore, } \\
\text { Amavita, Capitol, } \\
\text { Pharmacie Plus) }\end{array}$ & $17(30 \%)$ & $18(38 \%)$ & $124(49 \%)$ \\
\hline $\begin{array}{l}\text { Other pharmacy } \\
\text { type }\end{array}$ & $2(4 \%)$ & $0(0 \%)$ & $0(0 \%)$ \\
\hline \multicolumn{4}{|l|}{ Pharmacy location } \\
\hline Rural & $13(23 \%)$ & $6(13 \%)$ & NA \\
\hline Urban & $26(47 \%)$ & $25(53 \%)$ & NA \\
\hline Semi-urban & $17(30 \%)$ & $16(34 \%)$ & NA \\
\hline \multicolumn{4}{|l|}{ Pharmacy size } \\
\hline $\begin{array}{l}\text { Small }(<100 \\
\text { patients/day })\end{array}$ & $11(20 \%)$ & $19(40 \%)$ & NA \\
\hline $\begin{array}{l}\text { Medium (100-200 } \\
\text { patients/day) }\end{array}$ & $24(43 \%)$ & $18(38 \%)$ & NA \\
\hline $\begin{array}{c}\text { Large }(>200 \\
\text { patients/day) }\end{array}$ & $21(38 \%)$ & $10(22 \%)$ & NA \\
\hline \multicolumn{4}{|c|}{ Pharmacist participation in } \\
\hline Quality circles & $15(27 \%)$ & $8(17 \%)$ & NA \\
\hline $\begin{array}{l}\text { Current chronic } \\
\text { disease } \\
\text { management } \\
\text { programs }\end{array}$ & $18(32 \%)$ & $17(36 \%)$ & NA \\
\hline $\begin{array}{l}\text { Other non-specified } \\
\text { program }\end{array}$ & $6(11 \%)$ & $9(19 \%)$ & NA \\
\hline None & $17(30 \%)$ & $13(28 \%)$ & NA \\
\hline
\end{tabular}

NA not available

a 37 non-participating pharmacies did not provide information regarding their practice

\section{Discussion}

Recruitment of diabetic patients by pharmacies was feasible and satisfactory. Even if we did not reach our 600 targeted patients, this did not modify the precision of our point estimates because we got a larger number of clusters (pharmacies) and our dichotomous outcomes estimates had been conservative. This lower recruitment rate might be explained both by pharmacy- and patient-related barriers. In fact, more patients than expected were ineligible because they did not master French well enough, possibly presented cognitive impairment, or were not residing in the canton. Despite clear recruitment recommendation and frequent contacts with pharmacists, it remains unclear, however, how eligibility criteria were actually interpreted and applied, and whether patients were truly ineligible or 
Fig. 1 Recruitment flow diagram (Canton of Vaud, Switzerland, 2011)

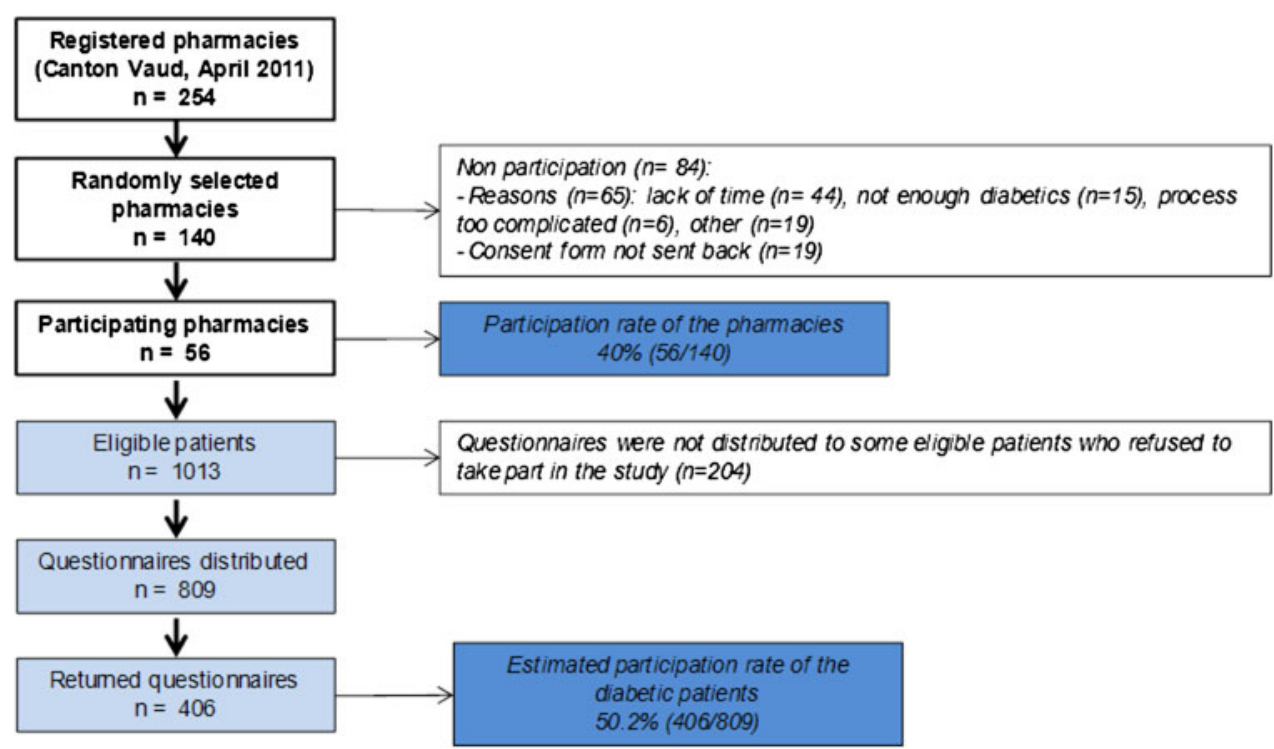

whether pharmacists "unintentionally applied selection criteria" (Knoester et al. 2005).

One important issue with this type of recruitment is the representativeness of such a sample. When comparing our sample to 260 known and treated diabetic patients aged 35-75 years, participating in a population-based cohort of residents of Lausanne (CoLaus), main town of the canton of Vaud, we noticed that they were rather similar on a few comparable variables (women 41 vs. $33 \%$, mean age 64 vs. 61 years, tertiary level education 25 vs. $20 \%$, current smokers 16 vs. $23 \%$, overweight and obesity 82 vs. $84 \%$, in our sample vs. the CoLaus sample, respectively) (P. Marques-Vidal, personal communication). Slight differences might be accounted for by differences in age ranges of the target population. This shows that in a same region, two recruitment strategies led to similar samples of diabetic participants. This similarity speaks against a major representativeness bias. However, both samples include only patients willing to participate in studies.

We chose this form of recruitment to involve physicians and pharmacists in the survey procedure, to minimize selection bias of both participating physicians and patients, to include patients treated by a variety of physicians and to limit the burden of physicians known to be very busy. In addition, our multidisciplinary working group hypothesized that pharmacist would be more ready to participate in the recruitment of patients than physicians. With regard to the latter reason, Swiss examples of diabetes screening projects in community pharmacies were promising (Bovet et al. 2011; Hersberger et al. 2006). Indeed, the recent pharmacy-based screening and awareness campaign for diabetes and cardiovascular risk factors in the canton of Vaud showed that, in 2010, $78 \%$ of registered pharmacies agreed to participate, allowing 4,222 individuals to be screened (Bovet et al. 2011). In that example, however (and others in the literature), pharmacists were actively participating in the intervention. They were not only asked to recruit patients and distribute questionnaires, but were actually assessing several chronic diseases risk factors and behaviors.

Informing, inciting and training pharmacists to take part in clinical research projects, prolonging and carefully monitoring the recruitment period, finding incentives to increase the pharmacists' and patients' motivation to participate could improve the yield of this recruitment method. In fact, participation in an intervention, where pharmacists would not only recruit patients but be actively involved in patients care, may be an interesting alternative. It may match pharmacists' interest to be further involved in new roles and help them overcome time constraints.

Easing recruitment of patients in primary care settings is needed. Pharmacy-based recruitment represents a valuable option that should be further developed and tested, particularly, since there is increasing evidence that pharmacists may help manage chronic diseases (McLean et al. 2008; Santschi et al. 2011).

Acknowledgments We would like to thank all pharmacies, diabetic patients and practicing physicians who participated in the study. We also thank the partners and members of the working group for their collaboration. We thank Mrs. Lucienne Boujon for copy editing the manuscript. This study was funded by the Department of Public Health of the canton of Vaud. Dr. I. Peytremann-Bridevaux is supported by a grant from the Swiss National Science Foundation [PROSPER $\mathrm{N}^{\circ}$ 32333B-123817 and $\mathrm{N}^{\circ}$ 32333B-139789].

Conflict of interest None of the authors has conflict of interest to declare. 


\section{References}

Bovet P, Hirsiger P, Emery F, De Bernardini J, Rossier C, Trebeljahr J, Hagon-Traub I (2011) Impact and cost of a 2-week community-based screening and awareness program for diabetes and cardiovascular risk factors in a Swiss canton. Diabetes Metab Syndr Obes 4:213-223

Hagon-Traub I, Hirsiger P, Bovet P, Ruiz J, Peytremann-Bridevaux I, Noth C, Hauschild (2010) Programme cantonal Diabète, présentation du programme. Lausanne: Service de la Santé Publique du canton de Vaud. http://www.vd.ch/fileadmin/user upload/themes/sante_social/services_soins/Diabete/fichiers_pdf/ Programme_cantonal.pdf

Hersberger KE, Botomino A, Mancini M, Bruppacher R (2006) Sequential screening for diabetes-evaluation of a campaign in Swiss community pharmacies. Pharm World Sci 28:171-179

Knoester PD, Belister SV, Deckers CLP, Keyser A, Renier WO, Egberts ACG, Hekster YA (2005) Recruitment of a cohort of lamotrigine users through community pharmacists: differences between patients who gave informed consent and those who did not. Pharmacoepidemiol Drug Saf 14:107-112
McLean DL, McAlister FA, Johnson JA, King KM, Makowsky MJ, Jones CA, Tsuyuki RT, SCRIP-HTN Investigators et al (2008) A randomized trial of the effect of community pharmacist and nurse care on improving blood pressure management in patients with diabetes mellitus: study of cardiovascular risk intervention by pharmacists-hypertension (SCRIP-HTN). Arch Intern Med 168:2355-2361

Nolte E, Knai C, McKee M (2008) Managing chronic conditions: experience in eight countries. World Health Organization on behalf of the European Observatory on Health Systems and Policies, Copenhagen

Santschi V, Chiolero A, Burnand B, Colosimo AL, Paradis G (2011) Impact of pharmacist care in the management of cardiovascular disease risk factors: a systematic review and meta-analysis of randomized trials. Arch Intern Med 171:1441-1453

Van Wieren-de Wijer DB, Maitland-van der Zee AH, de Boer A, Stricker BH, Kroon AA, de Leeuw PW, Bozkurt O, Klungel OH et al (2009) Recruitment of participants through community pharmacies for a pharmacogenetic study of antihypertensive drug treatment. Pharm World Sci 31:158-164 